\title{
MORFOLOGÍA POLÍNICA DE LACTUCEAE (ASTERACEAE) EN LA PENÍNSULA IBÉRICA. I. LACTUCA L. Y GÉNEROS RELACIONADOS
}

\author{
Emma MORENO-SOCÍAS, José Antonio MEJÍAS y María Josefa DÍEZ
}

\begin{abstract}
RESUMEN. Morfología polínica de Lactuceae (Asteraceae) en la Península Ibérica. I. Lactuca L. y géneros relacionados. Se estudia la morfología del polen de los taxones incluidos en los géneros Lactuca L., Prenanthes L., Cicerbita Wallr. y Mycelis Cass. que habitan en la Península Ibérica con los microscopios óptico (MO) y electrónico de barrido (MEB). El polen, 3-zonocolporado y equinolofado (sin lagunas polares), se caracteriza por presentar una cresta ecuatorial y 15 lagunas: 3 porales, 6 abporales y 6 paraporales. Tamaño pequeño o mediano, $\mathrm{P}$ x E $=21-41 \times 24-47 \mu \mathrm{m}$. Exina de 3-8 $\mu \mathrm{m}$ de grosor y ornamentación perforada y equinulado-equinada. Se han observado diferencias en cuanto al tamaño, amplitud de las apocolpias y tamaño de las espínulas-espinas. Nuestros resultados muestran de una manera bastante clara la afinidad entre los distintos géneros. En el caso de Lactuca, el género con mayor número de especies, los resultados polínicos permiten discutir sobre las relaciones evolutivas entre los representantes de la Península.
\end{abstract}

Palabras clave. Polen, taxonomía, harmomegatia, Lactuca, Prenanthes, Cicerbita, Mycelis.

SUMMARY. Pollen morphology of Lactuceae (Asteraceae) in the Iberian Peninsula. I, Lactuca L. and related genera. The pollen morphology of the taxa belonging to the genera Lactuca L., Prenanthes L., Cicerbita Wallr. y Mycelis Cass. in the Iberian Peninsula has been studied with light and electron microscopy. The pollen is 3-zonocolporate and echinolophate (without polar lacunae), with equatorial ridges and 15 lacunae: 3 poral, 6 abporal and 6 paraporal. Small to medium size, $\mathrm{P} \times \mathrm{E}=21-41 \times 24-47 \mu \mathrm{m}$. Exine 3-8 $\mu \mathrm{m}$ thick and ornamentation perforate and equinulate-equinate. Some differences in size, apocolpy size and spinules-spines size have been observed. The results clearly show the relationships between genera. The evolutionary significance of pollen morphology is discussed in the genus Lactuca which has the largest number of species in the Iberian Peninsula.

Key words. Pollen, taxonomy, harmomegathy, Lactuca, Prenanthes, Cicerbita, Mycelis.

\section{INTRODUCCIÓN}

En la familia Asteraceae, y dentro de la tribu Lactuceae, los géneros Lactuca L., Prenanthes L., Cicerbita Wallr. y Mycelis Cass. constituyen un grupo de géneros estrechamen- te relacionados e integrados, según Stebbins (1953), en una misma línea evolutiva independiente dentro de la tribu. De ellos, Prenanthes es considerado el género más primitivo. Se caracteriza por presentar cipselas columnares, cilíndricas o ligeramente comprimidas y sin 
pico. Su centro de origen se localiza en Asia Central y su distribución es fundamentalmente euroasiática y norteamericana. Cicerbita es un género de distribución exclusivamente euroasiática, intermedio entre Prenanthes y Lactuca, que presenta cipselas de oblongas a elípticas en las que se puede apreciar la tendencia general del grupo hacia la compresión del fruto y adquisición de un pico. Lactuca es el género más diversificado y extendido, con algunos taxones que se comportan como especies invasoras. Todos sus representantes muestran cipselas comprimidas y un pico muy marcado. Por último, Mycelis es un pequeño género distribuido por el Hemisferio Norte morfológicamente semejante a Lactuca. Se diferencia de éste por la disposición de las brácteas del involucro.

La Cuenca Mediterránea constituye un centro de diversificación, si no de origen, del género Lactuca (Feráková, 1977; Mejías, 1993). Está representado en la Península Ibérica por un total de diez taxones: L. viminea (L.) J. \& C. Presl subsp. viminea, L. viminea subsp. chondrilliflora (Boreau) Bonnier, $L$. viminea subsp. ramosissima (All.) Bonnier, $L$. serriola L., L. saligna L., L. virosa L., $L$. livida Boissier \& Reuter, L. perennis L. subsp. perennis, L. perennis subsp. granatensis Charpin \& Fernández Casas y L. tenerrima Pourret. De ellas, L. livida es endémica de la Península Ibérica y L. tenerrima de la Cuenca Mediterránea Occidental. El resto de los géneros, por el contrario, están representados por un total de sólo cuatro taxones: Prenanthes purpurea L., Cicerbita alpina (L.) Wallr., C. plumieri (L.) Kirschleger y Mycelis muralis (L.) Dumort.

La peculiar morfología del polen de las Lactuceae ha llevado a algunos autores a estudiar aspectos particulares del mismo, como las lagunas (Wodehouse, 1935; El-Ghazali, 1980; Blackmore, 1982a), las aberturas (Dimon, 1971; Blackmore, 1982b; Blackmore y Barnes, 1984) o la estructura y función de la exina
(Payne, 1981; Haque y Godward, 1983; Blackmore y Barnes, 1986). Estudios generales sobre la morfología del polen de los géneros mencionados anteriormente u otros relacionados han sido realizados por autores como Erdtman (1952), Ikuse (1956; 1962), Stix (1960), Wang (1960), Saad (1961), Davis y Raven (1962), Chaubal y Deodikar (1965), Nair (1965), Dimon (1971), Huang (1972), Kuprianova y Alyoshima (1972), Pons y Boulos (1972), Tomb (1975), Chaubal (1976), Skvarla et al. (1977), Parveen y Bhandari (1981), Blackmore (1981; 1982c; 1984), Díaz de la Guardia y Blanca (1983), Nair y Lawrence (1985), Gupta y Sharma (1986) y Díez (1987).

El propósito de este trabajo es, por un lado, aportar nuevos conocimientos sobre los taxones de Lactuca, Prenanthes, Mycelis y Cicerbita en la Península Ibérica y, por otro, ayudar a establecer la afinidad entre los distintos géneros. Por último, en el caso de Lactuca, el género con mayor número de especies y con un centro de diversificación en la Cuenca Mediterránea, discutir sobre las relaciones evolutivas entre los representantes de la Península Ibérica.

\section{MATERIAL Y MÉTODOS}

Se han estudiado 34 poblaciones cuya procedencia se indica en el Apéndice. Se ha utilizado tanto material de Herbario, del Departamento de Biología Vegetal y Ecología de la Universidad de Sevilla (SEV), como material fresco recolectado directamente en el campo e incluído en alcohol de $70^{\circ}$. En ambos casos las muestras se conservan en la Palinoteca del mismo Departamento.

El polen fue acetolizado por el método tradicional de Erdtman (1960).

Para cada muestra se efectuaron 30 medidas de los valores del eje polar (P) y diámetro ecuatorial (E), y 10 para el resto de los caracteres: grosor de la exina, sexina y nexina en la 
mesocolpia, endoaberturas, mesoaberturas, amplitud del casquete polar y tamaño de los elementos supratectales.

El estudio de la ornamentación se ha llevado a cabo con un microscopio electrónico de barrido (MEB). Para ello, el polen, una vez deshidratado en la serie de alcoholes, se colocó en el portaobjetos, procediéndose posteriormente a su metalización con oro-paladio y observación a $25 \mathrm{kv}$.

Las fotografías al microscopio óptico (MO) se realizaron en un fotomicroscopio marca NIKON y las del MEB en un microscopio marca JEOL modelo JSM-T 100.

Para las descripciones polínicas se ha seguido fundamentalmente la terminología de Wodehouse (1935), Stix (1960) y Dimon (1971).

\section{RESULTADOS}

El polen de Lactuca y géneros relacionados es isopolar radiosimétrico, 3zonocolporado (sistema apertural tripartito; figs. 3,11 ) y equinolofado (sin lagunas polares), con una cresta ecuatorial (fig. 10) y 15 lagunas: 3 porales, 6 abporales y 6 paraporales delimitadas por crestas y con casquete polar más o menos amplio (figs. 12-14). Sobre el casquete y las crestas aparecen espínulas 0 espinas de 1-6 $\mu \mathrm{m}$ de altura; la base de las espínulas/espinas, así como la superficie de las crestas y el casquete se encuentran perforado-finamente reticuladas (fig. 15). El tamaño es pequeño o mediano, $\mathrm{P}$ x E = 21-41 x 24-47 $\mu \mathrm{m}$. La exina, de 3-8 $\mu \mathrm{m}$ de grosor presenta la sexina más gruesa que la nexina y el infratectum columelado. En general es equiaxo, de contorno circular en visión ecuatorial y corte óptico meridiano (c.o.m.; fig. 1) y hexagonal en visión polar y corte óptico ecuatorial (c.o.e.; figs. 4, 5).

Las medidas de los caracteres cuantitativos del polen de los taxones estudiados se recogen en la tabla 1 . Se ha observado variación en el tamaño del polen y las espinas, así como en la amplitud del casquete polar. Estas diferencias nos permiten establecer cuatro grupos:

GRUPO I. Incluye Cicerbita alpina, $C$. plumieri y Prenanthes purpurea (figs. 1, 3, 4, 6).

Se caracteriza por presentar las mayores dimensiones, tanto de los ejes: $\mathrm{P} \times \mathrm{E}=$ $28(X \geq 35.1) 41 \times 32(X \geq 38.7) 47 \mu \mathrm{m}$, como del casquete polar: (10-)11-18 (-19) $(\mathrm{X} \geq 11.4)$ $\mu \mathrm{m}$ y las espinas: $3-6(\mathrm{X} \geq 4.1) \mu \mathrm{m}$ de altura.

GRUPO II. Incluye Lactuca perennis subsp. granatensis y L. perennis subsp. perennis (figs. 7, 12).

El tamaño de los ejes es algo menor que en el grupo anterior: $\mathrm{P} x \mathrm{E}=25(\mathrm{X} \leq 32.1) 40$ $x 27(X \leq 35.9) 46 \mu \mathrm{m}$. La amplitud del casquete polar es similar a la de Cicerbita alpina, (8) 10-13 $(-14,-15)(X \leq 11.8) \mu \mathrm{m}$. Las espínulas/ espinas miden $2-3(\mathrm{X} \leq 2.3) \mu \mathrm{m}$ de altura.

GRUPO III. Incluye L. livida, L. saligna, $L$. serriola, L. tenerrima, L. virosa y Mycelis muralis (figs. 2, 5, 9, 11, 13, 15).

Presenta, junto al Grupo IV, las menores dimensiones polínicas: $\mathrm{P} \times \mathrm{E}=22(\mathrm{X} \leq 29.8)$ 36 × $24(\mathrm{X} \leq 32.7) 40 \mu \mathrm{m}$. La amplitud del casquete polar es más pequeña que en los grupos anteriores: $5-11(\mathrm{X} \geq 5.9) \mu \mathrm{m}$. Las espínulas miden $2-2.5(-3)(\mathrm{X} \leq 2.1) \mu \mathrm{m}$.

GRUPO IV. Incluye Lactuca viminea subsp. chondrilliflora, L. viminea subsp. ramosissima y L. viminea subsp. viminea (figs. 8, 10, 14).

Las dimensiones polínicas son similares a las del grupo anterior: $\mathrm{P} \times \mathrm{E}=21(\mathrm{X} \leq 29.3)$ $34 \times 25(\mathrm{X} \leq 31.4) 36 \mu \mathrm{m}$. La amplitud del casquete polar es la más pequeña: $3-5.5(-6)(\mathrm{X}$ $\leq 4.5) \mu \mathrm{m}$. Las espínulas miden $1-2.5(\mathrm{X} \leq 2.1)$ $\mu \mathrm{m}$. 

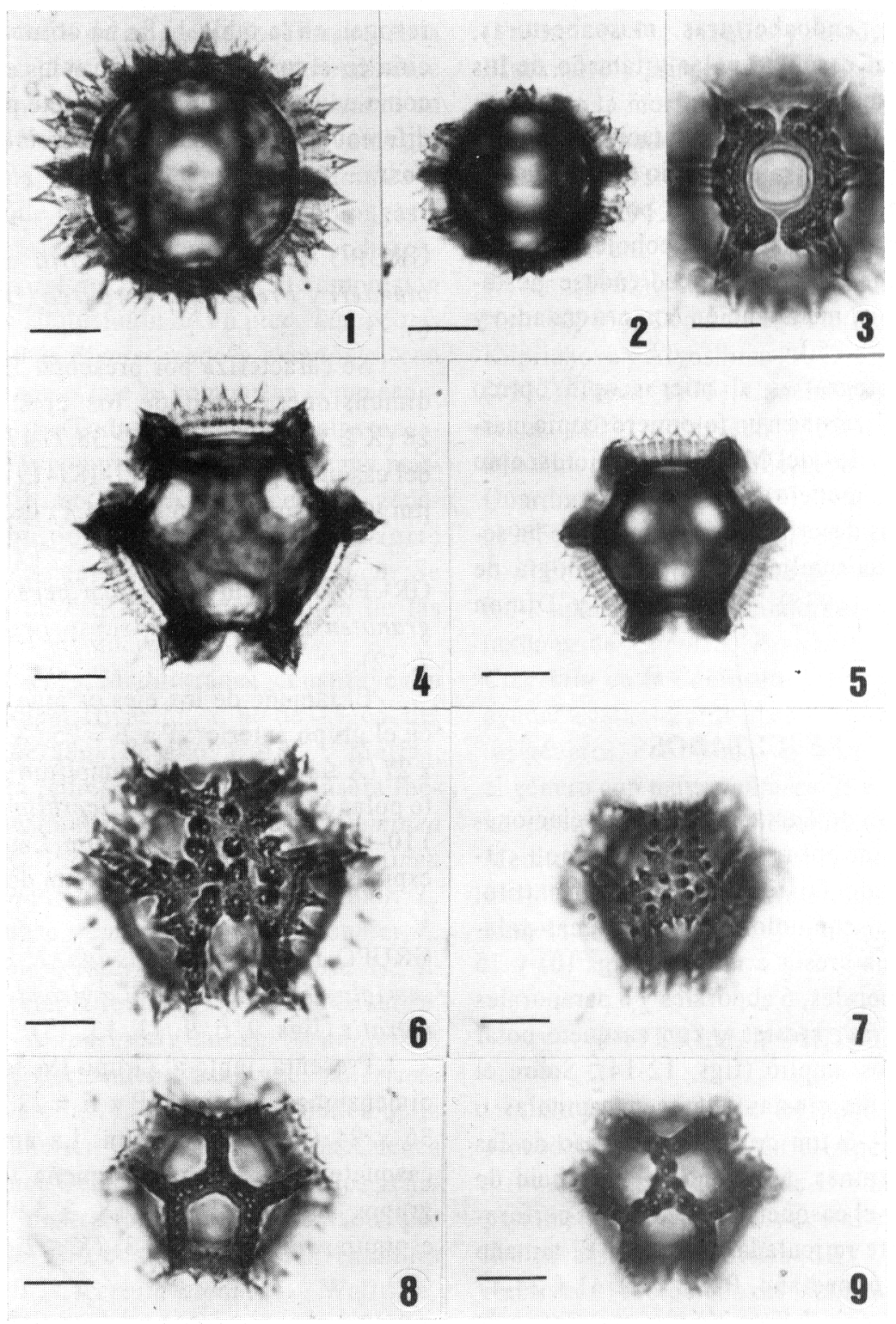

Figuras 1-9. 1)Prenanthes purpurea, visión ecuatorial en c.o.m. 2)Lactuca serriola, visión ecuatorial en c.o.m. 3)Cicerbita plumieri, abertura. 4)Cicerbita plumieri, visión polar en c.o.e. 5)Lactuca serriola, visión polar en c.o.e. 6)Prenanthes purpurea, apocolpia. 7)Lactuca perennis subsp.'granatensis, apocolpia. 8)Lactuca viminea subsp. chondrilliflora, apocolpia. 9)Lactuca serriola, apocolpia. Escala: $10 \mu \mathrm{m}$.

1) Prenanthes purpurea, outline in equatorial view, optical section. 2)Lactuca serriola, outline in equatorial view, optical section. 3)Cicerbita plumieri, aperture. 4)Cicerbita plumieri, outline in polar view, optical section. 5)Lactuca serriola, outline in polar view, optical section. 6)Prenanthes purpurea, apocolpium. 7)Lactuca perennis subsp. granatensis, apocolpium. 8)Lactuca viminea subsp. chondrilliflora, apocolpium. 9)Lactuca serriola, apocolpium. Scale bars: $10 \mu \mathrm{m}$. 

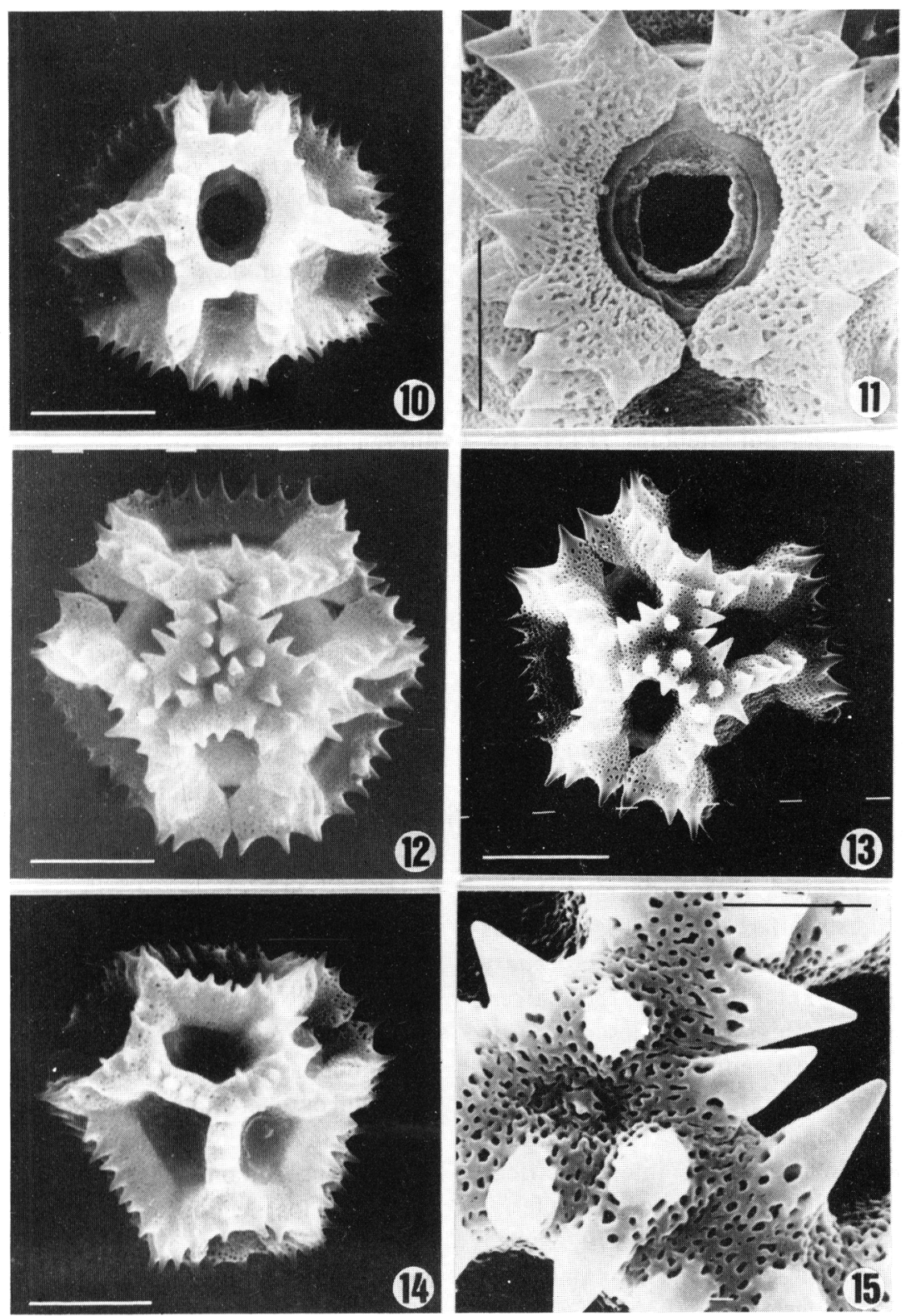

Figuras 10-15. 10)Lactuca viminea subsp. chondrilliflora, visión ecuatorial. 11)Mycelis muralis, detalle de la abertura. 12)Lactuca perennis subsp. granatensis, visión polar. 13)Lactuca tenerrima, visión polar. 14)Lactuca viminea subsp. chondrilliflora, visión polar. 15)Lactuca tenerrima, detalle de la ornamentación en la apocolpia. Escala: Figs. $10-14=10 \mu \mathrm{m}$; fig. $15=5 \mu \mathrm{m}$.

10)Lactuca viminea subsp. chondrilliflora, equatorial view. 11)Mycelis muralis, detail of aperture. 12)Lactuca perennis subsp. granatensis, polar view. 13)Lactuca tenerrima, polar view. 14)Lactuca viminea subsp. chondrilliflora, polar view. 15)Lactuca tenerrima, apocolpium, detail of ornamentation. Scale bars: figs. $10-14=10 \mu \mathrm{m}$; fig. $15=5 \mu \mathrm{m}$ 


\section{DISCUSIÓN}

Como se observa en los resultados, el estudio de los caracteres del polen de los taxones analizados permite la separación de cuatro grupos que ponen de manifiesto un cierto interés taxonómico. Estos grupos se diferencian en tres caracteres: tamaño de los ejes, altura de las espínulas/espinas y amplitud del casquete polar. Curiosamente, se observa una disminución progresiva en dichos caracteres que coincide con la línea evolutiva PrenanthesLactuca propuesta por Stebbins (1953; fig. 16).

En general, el tamaño de los granos de polen en Angiospermas parece ser un carácter con importante grado de variabilidad intraespecífica, dependiente de diversos factores del medio físico, como la nutrición mineral y/o la disponibilidad de agua (SchochBodmer, 1940; Walker y Doyle, 1975; Walker, 1976; Müller, 1979; Ojeda y Díez, 1992) y las características climáticas (Lobreau-Callen, 1975). Sin embargo, la constancia en los datos obtenidos parece indicar una disminución efectiva de este carácter con la evolución del grupo. Tendencias similares han sido observadas en Boraginaceae (Díez, 1981), Saruraceae y Piperaceae (Walker y Doyle, 1975; Walker, 1976), aunque también se han descrito casos en sentido inverso, como en Annonaceae (Walker y Doyle, 1975), no existiendo ningún patrón fijo en Angiospermas. Algunos autores han relacionado diferencias interespecíficas encontradas en algunos grupos con características del sistema sexual (Müller, 1979; Cruden y Lyon, 1985) o con condicionamientos climáticos y geográficos (Lobreau-Callen, 1975) y, en el presente caso, se observa un menor tamaño en los representantes de áreas más cálidas con importante incidencia de autofecundación (Mejías, en prensa). Por otro lado, es sobradamente conocida la relación entre tamaño del polen y dotación cromosómica; sin embargo, en ningún caso las diferencias interespecíficas encontradas en los taxones estudiados están relacionadas con diferencias en la dotación cromosómica, pues todos presentan el número $2 \mathrm{n}=18$, excepto Cicerbita plumieri y Lactuca tenerrima con $2 n=16$, y se desconoce la existencia de diferentes niveles de ploidía (Mejías, 1993).

En lo referente a las variaciones en longitud de espínulas/espinas entre taxones, no parece haber ninguna explicación. Tampoco se ha encontrado en la bibliografía consultada ninguna hipótesis que trate de explicar tales diferencias.

Respecto a la disminución del casquete polar, el hecho supone un aumento de la superficie de las lagunas en el grano de polen. Blackmore (1982a) encontró en la tribu Lactuceae una fuerte correlación entre el aumento de la superficie lagunar y el desarrollo de elaboradas formas de la función harmomegática, siendo ésta especialmente significativa cuando las lagunas son extensas y las aberturas están inmovilizadas por crestas ecuatoriales (Blackmore, 1982a; Blackmore y Barnes, 1986). Esto representa niveles de adaptación variables a las condiciones xéricas o de escasa disponibilidad hídrica. La adaptación en polen equinolofado con crestas ecuatoriales, como el presente, sería máxima en los casos en que el casquete se reduce a la intersección de las crestas. La variación de la amplitud del casquete polar en el grupo estudiado está relacionado con su expansión evolutiva; así, los representantes más primitivos, pertenecientes a los géneros Prenanthes y Cicerbita y que ocupan herbazales y bosques de altitud con clima frío y corto período de crecimiento estival en que el agua no suele ser factor limitante, muestran casquetes polares de gran amplitud. Por el contrario, el género Lactuca, que incluye los representantes más evolucionados del grupo (Stebbins 1953, Stebbins et al. 1953, Mejías 1993), se ha extendido en la Región Mediterránea desde zonas montañosas a áreas de menor altitud manteniendo el perío- 


\begin{tabular}{|c|c|c|c|c|c|}
\hline & $P$ & $\mathrm{E}$ & casquete & $\begin{array}{l}\text { espínulas/ } \\
\text { espinas }\end{array}$ & exina \\
\hline \multicolumn{6}{|l|}{ Grupo I } \\
\hline C. alpina & $\begin{array}{l}29-40 \\
(36.4)\end{array}$ & $\begin{array}{l}32-42 \\
(38.7)\end{array}$ & $\begin{array}{l}(10) 11-12 \\
(11.4)\end{array}$ & $\begin{array}{c}4-5 \\
(4.2)\end{array}$ & $5-7$ \\
\hline C. plumieri & $\begin{array}{l}28-40 \\
(35.1)\end{array}$ & $\begin{array}{l}32-42 \\
(38.8)\end{array}$ & $\begin{array}{l}15-18(19) \\
(16.4)\end{array}$ & $\begin{array}{l}3.5-6 \\
(4.2)\end{array}$ & $5-7$ \\
\hline P. purpurea & $\begin{array}{l}28-41 \\
(35.6)\end{array}$ & $\begin{array}{l}32-47 \\
(40.1)\end{array}$ & $\begin{array}{c}(12-13) 15-17(18) \\
(15.2)\end{array}$ & $\begin{array}{c}3-6 \\
(4.1)\end{array}$ & $5-6(8)$ \\
\hline \multicolumn{6}{|l|}{ Grupo II } \\
\hline $\begin{array}{l}\text { L. perennis } \\
\text { subsp. granatensis }\end{array}$ & $\begin{array}{l}26-33 \\
(29.0)\end{array}$ & $\begin{array}{l}28-39 \\
(33.1)\end{array}$ & $\begin{array}{l}10-13(15) \\
\quad(11.8)\end{array}$ & 2 & $5-6$ \\
\hline $\begin{array}{l}\text { L. perennis } \\
\text { subsp. perennis }\end{array}$ & $\begin{array}{l}25-40 \\
(32.1)\end{array}$ & $\begin{array}{l}27-46 \\
(35.9)\end{array}$ & $\begin{array}{c}(8) 10-13(14-15) \\
(11.7)\end{array}$ & $\begin{array}{l}2-3 \\
(2.3)\end{array}$ & (4) $5-6$ \\
\hline \multicolumn{6}{|l|}{ Grupo III } \\
\hline L. livida & $\begin{array}{l}28-31 \\
(29.3)\end{array}$ & $\begin{array}{l}29-35 \\
(32.7)\end{array}$ & $\begin{array}{c}(7) 8-9(10) \\
(8.3)\end{array}$ & 2 & $4-6$ \\
\hline L. saligna & $\begin{array}{l}22-31 \\
(26.9)\end{array}$ & $\begin{array}{l}24-35 \\
(29.6)\end{array}$ & $\begin{array}{l}5-7(8) \\
(5.9)\end{array}$ & 2 & (4) $5-7$ \\
\hline L. serriola & $\begin{array}{l}25-30 \\
(27.2)\end{array}$ & $\begin{array}{l}28-34 \\
(30.3)\end{array}$ & $\begin{array}{l}5-8(9) \\
(6.8)\end{array}$ & 2 & $4-5(6)$ \\
\hline L. tenerrima & $\begin{array}{l}25-32 \\
(28.3)\end{array}$ & $\begin{array}{l}26-36 \\
(31.6)\end{array}$ & $\begin{array}{l}(7) 8-9(10) \\
(8.3)\end{array}$ & $\begin{array}{l}2(3) \\
(2.1)\end{array}$ & $4-5$ \\
\hline L. virosa & $\begin{array}{l}26-34 \\
(29.5)\end{array}$ & $\begin{array}{l}28-35 \\
(32.3)\end{array}$ & $\begin{array}{c}(6) 7-11 \\
(8.8)\end{array}$ & $\begin{array}{l}1.5-3 \\
(2.0)\end{array}$ & (3) $4-5(6)$ \\
\hline M. muralis & $\begin{array}{l}24-36 \\
(29.8)\end{array}$ & $\begin{array}{l}26-40 \\
(32.3)\end{array}$ & $\begin{array}{l}5-10 \\
(7.5)\end{array}$ & $\begin{array}{l}2-2.5(3) \\
(2.1)\end{array}$ & (4) $5-6$ \\
\hline \multicolumn{6}{|l|}{ Grupo IV } \\
\hline $\begin{array}{l}\text { L. viminea } \\
\text { subsp. chondrilliflora }\end{array}$ & $\begin{array}{l}21-34 \\
(28.2)\end{array}$ & $\begin{array}{c}25-36 \\
(31)\end{array}$ & $\begin{array}{l}3.5-5(6) \\
(4.5)\end{array}$ & $\begin{array}{c}1-2 \\
(1.6)\end{array}$ & $5-7$ \\
\hline $\begin{array}{l}\text { L. viminea } \\
\text { subsp. ramosissima }\end{array}$ & $\begin{array}{l}25-33 \\
(29.3)\end{array}$ & $\begin{array}{l}25-35 \\
(31.4)\end{array}$ & $\begin{array}{c}3.5-5.5 \\
(4.3)\end{array}$ & $\begin{array}{l}(1) 2-2.5 \\
(2.1)\end{array}$ & $5-6$ \\
\hline $\begin{array}{l}\text { L. viminea } \\
\text { subsp. viminea }\end{array}$ & $\begin{array}{l}23-31 \\
(28.1)\end{array}$ & $\begin{array}{l}26-34 \\
(30.3)\end{array}$ & $\begin{array}{c}3-5 \\
(4.2)\end{array}$ & $\begin{array}{l}2-2.5 \\
(2.1)\end{array}$ & $5-6(7)$ \\
\hline
\end{tabular}

Tabla 1. Valores medios y rangos encontrados, distribuidos por grupos morfológicos, de las medidas $(\mu \mathrm{m})$ de los ejes polar (P) y ecuatorial (E), amplitud del casquete, longitud de espínulas/espinas y grosor de la exina.

Mean and ranges $(\mu m)$, arranged in pollen groups for the polar $(P)$ and equatorial $(E)$ axes, apocolpy size, spinules-spines size and exine thick. 
do de desarrollo estival. Ha colonizado, de esta forma, áreas más cálidas con un ciclo estacional más prolongado pero menor disponibilidad de agua (dentro de este último caso podemos citar $L$. serriola y $L$. saligna que pueden encontrarse incluso colonizando suelos salobres). Paralelamente, se aprecia una reducción progresiva del casquete polar que en su fase final está constituido por la intersección de las crestas, lo que parece permitir el máximo desarrollo de la función harmomegática.

Muy interesantes son los resultados obtenidos en los taxones Lactuca perennis y $L$. tenerrima, muy emparentados. El primero es una especie mediterránea de áreas montañosas con veranos frescos y disponibilidad de agua, siendo su casquete polar el mayor encontrado en el género. L. tenerrima forma también parte de la flora montañosa mediterránea, pero soporta temperaturas estivales mucho más extremas y mayor déficit hídrico, presentando un casquete de amplitud menor. Una relación semejante puede observarse entre $L$. virosa, $L$. livida y $L$. serriola, taxones nitrófilos con biología semejante y claramente emparentados; los dos primeros colonizan áreas de veranos relativamente suaves, mientras $L$. serriola ocupa zonas más cálidas. Consecuentemente, la última muestra un casquete más reducido.

AGRADECIMIENTOS. Los autores desean expresar su agradecimiento a Edinburgh University Press, Birkhäuser Verlarg y Ketrés Editora por permitir el uso de material gráfico de sus obras The Flora of Turkey 5: 781, Flora der Schweiz 3: 627 y 634 y Flora Vascular de Andalucía Occidental 3: 83 y 85 , respectivamente, para la figura 16 ; y a M. Martín Cacao por la realización de los dibujos de granos de polen.

\section{APÉNDICE}

Cicerbita alpina (L.) Wallr.

GERONA. Mejías, Polo \& Romero (SEV 132117).
Cicerbita plumieri (L.) Kirschleger.

ASTURIAS. Barrera \& Mejías (SEV 126404). LÉRIDA. Mejías, Polo \& Romero (SEV 126409). SORIA. Segura Subizarreta (SEV 41090).

Lactuca livida Boiss. \& Reuter.

TOLEDO. Arroyo \& Mejías (SEV 124612).

Lactuca perennis L. subsp. granatensis Charpin \& Fernández Casas.

GRANADA. Cabezudo \& Talavera (SEV 56554).

Lactuca perennis L. subsp. perennis

BURGOS. Díaz, Santabárbara \& Vioque (SEV 126577). GERONA. Devesa, Luque \& Romero (SEV 63839). LÉRIDA. Díaz, López \& Pino (SEV 126544).

Lactuca saligna $\mathrm{L}$.

CÁDIZ. Herrera, Mejías \& Muñoz (SEV 126629). ÁLAVA. García \& Mejías (SEV 118394). ALBACETE. Mejías, Polo \& Romero (SEV 126533).

Lactuca serriola $\mathrm{L}$.

ALBACETE. Mejías, Polo \& Romero (SEV 133574). BARCELONA. Mejías, Polo \& Romero (SEV 133573).

Lactuca tenerrima Pourret

JAÉN. Herrera \& Mejías (SEV 124653). LEÓN. García \& Mejías (SEV 124663).

Lactuca viminea subsp. chondrilliflora (Boreau) Bonnier

GERONA. Mejías, Polo \& Romero (SEV 126380). Mejías, Polo \& Romero (SEV 126380). LÉRIDA. Mejías, Polo \& Romero (SEV 126382).

Lactuca viminea subsp. ramosissima (All.) Bonnier ASTURIAS. Barrera \& Mejías (SEV 126385). GRANADA. Mejías \& Talavera (SEV 126579). SEVILLA. Herrera, Mejías \& Moreno (SEV 126488). ZARAGOZA. Devesa, Gallego, Luque \& Talavera (SEV 54939).

Lactuca viminea (L.) J. \& C. Presl subsp. viminea (L.) J. \& C. Presl.

HUELVA. Mejías (SEV 126379). TOLEDO. Arroyo \& Mejías (SEV 124618). 

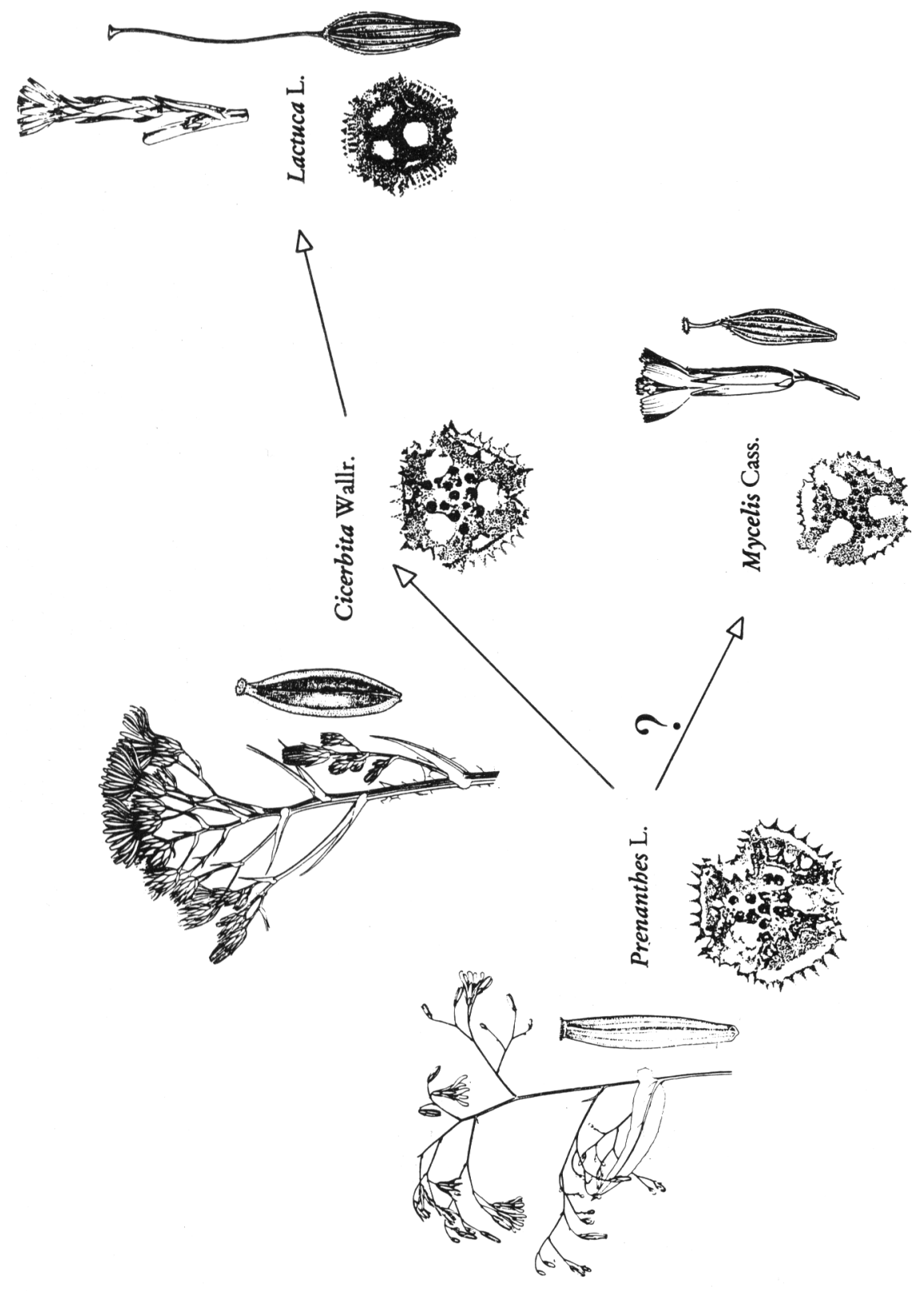

एक

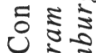
ब․ㅁㄴ. 寉 음 司. 등 욜 要 a

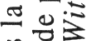

丞

芯志

몰

क ⿻ 큰 D \&

벌

凶ว

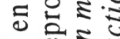

กิ

นู은

政造

药

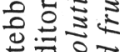

ज可焉

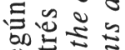

i 0

ㄴ.

娄主

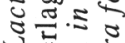

뒁

出年

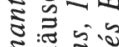

논

2.

的氛

清 0

므웡

을원

읜

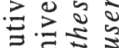

긍ㅎㅀ

यद्

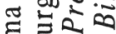

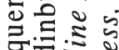

대되

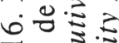

○

픕.

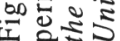


Lactuca virosa $\mathrm{L}$.

ALBACETE. Mejías \& Arroyo (SEV 124622).

ASTURIAS. Barrera \& Mejías (SEV 126567).

BARCELONA. Mejías. Polo \& Romero (SEV 126594).

Mycelis muralis (L.) Dumort

ALBACETE. Mejías, Polo \& Romero (SEV 126425). ASTURIAS. Barrera \& Mejías (SEV 126444). GERONA. Mejías, Polo \& Romero (SEV 126432).

Prenanthes purpurea $\mathrm{L}$.

GERONA. Mejías, Polo \& Romero (SEV 126413). HUESCA. Mejías (SEV 126588). LÉRIDA. Mejías, Polo \& Romero (SEV 126419).

\section{BIBLIOGRAFÍA}

BLACKMORE, S. -1981- Palynology and intergeneric relationships in the subtribe Hyoseridinae (Compositae: Lactuceae), Bot. J. Linn. Soc., 82: 1-13.

BLACKMORE, S. -1982a- A functional interpretation of Lactuceae (Compositae) pollen. Pl. Syst. Evol., 141: 153-168.

BLACKMORE, S. $-1982 \mathrm{~b}$ - The apertures of Lactuceae (Compositae) pollen. Pollen et Spores, 24: 453-462.

BLACKMORE, S. -1982c- Palynology of subtribe Scorzonerinae (Compositae: Lactuceae) and its taxonomic significance. Grana, 21: 149-160.

BLACKMORE, S. -1984- The Northwest European Pollen Flora, 32. Compositae-Lactuceae. Rev. Palaeobot. Palynol., 42: 45-85.

BLACKMORE, S. \& S.H. BARNES -1984- Low temperature scanning electron microscopy of pollen. Grana, 23: 157-162.

BLACKMORE, S. \& S.H. BARNES -1986Harmomegathic mechanisms in pollen grains. En S. BLACKMORE y I. K. FERGUSON (eds.): Pollen and spores: form and function. Linn. Soc. Symp. Ser. no 12: 137-149. Academic Press. London.

CHAUBAL, P.D. -1976- Palynological aspects of Compositae. Dept. Botany Shirajii Univ. Kdhapur, India.

CHAUBAL, P.D. \& G.B. DEODIKAR -1965- Pollen morphotypes in the family Compositae from parts of Western Ghats (India). Palynol. Bull. Lucknow, II y III: 84-88.

CRUDEN, R.W. \& D.L. LYON-1985- Correlations among stigma depth, style length, and pollen grain size: do they reflect function or phylogeny?. Bot. Gaz., 146: 143-149.

DAVIS, W.S. \& P.H. RAVEN -1962- Three new species related to Malacothrix clevelandii. Madroño, 16: 258-266.

DÍAZ DE LA GUARDIA, C. y G. BLANCA -1983. Morfología polínica del género Scorzonera L. (Asteraceae). En N. SOLÉ de PORTA y M. SUÁREZ CERVERA (eds.): Actas del IV Simposio de Palinología. Universitat de Barcelona. Barcelona. pp. 29-38.

DÍEZ, M.J. -1981-Estudio palinológico de las especies españolas de la familia Boraginaceae. Tesis doctoral. Universidad de Sevilla.

DÍEZ, M.J. -1987- Asteraceae (Compositae). En B. VALDÉS, M. J. DÍEZ e I. FERNÁNDEZ (eds.): Atlas polínico de Andalucía Occidental. Instituto de Desarrollo Regional de la Universidad de Sevilla y Excma. Diputación de Cádiz. Utrera (Sevilla). pp. 332-357.

DIMON, M.T. -1971- Problémes généraux soulevés par l'étude pollinique des Composées méditerranéennes. Naturalia Monspeliensia, sér. Bot., 22: 129-144.

EL-GHAZALI, G. -1980- Palynology of Hypochoeridinae and Scolyminae (Compositae). Opera Bot. 58: 1-48.

ERDTMAN, G. -1952- Pollen morphology and plant taxonomy. Angiosperms. (An introduction to palynology I). Almquist and Wiksell. Stockholm.

ERDTMAN, G. -1960- The acetolysis method. A revised description. Svensk. Bot. Tidskr. 54: 561-564.

FERÁKOVÁ, V. -1977- The genus Lactuca L. in Europe. Univerzita Komenského.

GUPTA, A. \& CH. SHARMA -1986- Pollen flora of North-West Himalaya. Indian Association of Palynostratigraphers. Lucknow, India.

HAQUE, M.Z. \& H.B.E. GODWARD -1983Evidence of genic control exine pattern from irradiation studies in Lactuceae. Pollen et Spores, 25: 421-436.

HUANG, T.C. -1972- Pollen flora of Taiwan. National Taiwan Univ., Botany Dept. Press.

IKUSE, M. -1956- Pollen grains of Japan. Hirokawa 
Publishing Co., Tokyo.

IKUSE, M. -1962- On pollen grains of the Compositae collected by the Kyoto University to the Karakoran and Hindukush 1955. Acta Phytotax. Geobot., 20: 112-119.

KUPRIANOVA, L.A. \& L.A. ALYOSHIMA - 1972Pollen and spores of plants from the flora of European part of USSR, vol. l. Acad. Sci. USSR. Komarov Bot. Inst.

LOBREAU-CALLEN, D. -1975- Les variations dimensionnelles du pollen du genre Ilex (Aquifoliaceae) et leurs rapports avec le climat. Bull. Soc. Bot. Fr., Coll. Palynologie, 122: 179-199.

MEJÍAS, J.A. -1993-Cytotaxonomic studies in the Iberian taxa of the genus Lactuca (Compositae). Bot. Helv., 103: 113-130.

MEJÍAS, J.A. - 1994- Self-fertility and flower heads traits in the Iberian taxa of Lactuca and related genera (Asteraceae, Lactuceae). Pl. Syst. Evol. (en prensa).

MÜLLER, J. -1979- Form and function in Angiosperm pollen. Ann. Missouri Bot. Gard., 66: 593-632.

NAIR, P.K.K. -1965- Pollen grains of Western Himalaya plants. Asia Monographs, India, 1: $1-102$.

NAIR, P.K.K. \& R. LAWRENCE -1985- Pollen morphology of Indian Compositae. Adv. in Pollen Spores Res., 14: 1-176.

OJEDA, F. \& M.J. DÍEZ - 1992- Pollen dimorphism in three Teucrium species (Lamiaceae). Pl. Syst. Evol., 183: 43-49.

PARVEEN F. \& M. M. BHANDARI -1981- Pollen morphology of desert Asteraceae. J. Palynol., 17: 121-130.

PAYNE, W.W. -1981- Structure and function in Angiosperm pollen wall evolution. Rev. Palaeobot. Palynol., 35: 39-59.

PONS, A. y L. BOULOS -1972-Révisión systématique du genre Sonchus L. s.l. III. Étude palynologique. Bot. Not., 125: 310-319.

SAAD, S.I. -1961- Pollen morphology in the genus Sonchus. Pollen et Spores, 3: 247-274.

SCHOCH-BODMER, H. -1940- The influence of nutrition upon pollen-grain size in Lythrum salicaria. J. Genet., 40: 393-402.

SKVARLA, J.J., B.L. TURNER, V.C. PATEL \& A.S. TOMB -1977- Pollen morphology in the Compositae and in morfologically related families. En V. H. HEYWOOD, J. B. HARBORNE y B. L. TURNER (eds.): The biology and Chemistry of the Compositae. Academic Press. London. pp. 141-265.

STEBBINS, G.L. -1953- A new classification of the Tribe Cichorieae family Compositae. Madroño, 12: 65-81.

STEBBINS, G.L., J.A. JENKINS \& M.S. WALTERS -1953- Chromosomes and phylogeny in the Compositae, tribe Cichorieae. Univ. Calif. Publ. Bot., 26: 401-430.

STIX, E. -1960- Pollen morphologische Untersuchungen an Compositen. Grana Palynol., 2: 41-104.

TOMB, A.S. -1975- Pollen morphology in Tribe Lactuceae (Compositae). Grana, 15: 79-89.

WALKER, J.W. -1976- Evolutionary significance of the exine in the pollen of primitive Angiosperms. En I. K. FERGUSON y J. MÜLLER (eds.): The evolutionary significance of the exine. Linn. Soc. Symp., ser. 1: 251-308.

WALKER, J.W. \& J.A. DOYLE - 1975- The bases of Angiosperm phylogeny: Palynology. Ann. Missouri Bot. Gard., 62: 664-723.

WANG, F.H. -1960- Pollen grains of China. Acta Bot. Sin., 4: 269-279.

WODEHOUSE, R. -1935- Pollen grains. Hafner Publishing Co., New York.

Aceptado para su publicación en Septiembre de 1993

Dirección de los autores. Departamento de Biología Vegetal y Ecología. Facultad de Biología. Apdo. 1095, 41080-Sevilla 